Check for updates

Cite this: Mater. Adv., 2020, 1,1788

Received 1st June 2020

Accepted 21st July 2020

DOI: $10.1039 / \mathrm{d} 0 \mathrm{ma00370k}$

rsc.li/materials-advances

\title{
Building thermally and chemically reversible covalent bonds in vegetable oil based epoxy thermosets. Influence of epoxy-hardener ratio in promoting recyclability $\dagger$
}

\author{
Chiara Di Mauro, ${ }^{a}$ Aratz Genua ${ }^{\mathrm{b}}$ and Alice Mija (D) *a
}

\begin{abstract}
Thermoset polymers with permanently cross-linked networks formed via thermally reversible covalent bonds have outstanding self-healing and adaptable properties, combined with very good mechanical properties and solvent resistance. This work reports for the first time the synthesis and characterization of such materials, derived from epoxidized linseed or soybean oils crosslinked with different amounts of $2,2^{\prime}$-dithiodibenzoic acid. The epoxy/hardener ratio was studied as a key parameter influencing the overall process and moreover the network recyclability. The synthesized thermosets showed excellent chemical recycling and mechanical reprocessing abilities. The produced networks were chemically recycled, then the recuperated reprocessed material was found to show no or a small decrease in mechanical properties. These biobased thermosets provide opportunities for the circular use of thermosets.
\end{abstract}

\section{Introduction}

Natural, biobased resources have been providing a myriad of possibilities in the last decade to produce new chemicals and materials. For this reason, the development of polymers derived from vegetable oils is steadily increasing, due to their low cost and lack of toxicity, in addition to the fact that they give access to processes minimizing $\mathrm{CO}_{2}$ emission, which is responsible for global warming. To avoid the continuous accumulation of materials in landfill sites, we should find and propose solutions, for example, for the production of materials, especially thermosets, with recyclability properties.

Vegetable oils (VOs) are an important family of renewable materials, mainly constituted by triglycerides formed from glycerol and fatty acids. ${ }^{1}$ The most common vegetable oils contain fatty acid chains of length from 14 to 22 carbon atoms. Many methods of double bond functionalization have been proposed $^{2}$ and much applied because they allow mild conditions and green processes in the oxidation of unsaturated molecules, producing epoxy derivatives.

Epoxidized vegetable oils (EVOs), mainly epoxidized linseed oil (ELO) and epoxidized soybean oil (ESO), are used in

\footnotetext{
${ }^{a}$ Université Côte d'Azur, Institut de Chimie de Nice, UMR 7272 - CNRS, 28 Avenue Valrose, 06108 Nice Cedex 2, France.E-mail: Alice.MIJA@univ-cotedazur.fr

${ }^{b}$ CIDETEC, Basque Research and Technology Alliance (BRTA), Paseo Miramón 196, Donostia-San Sebastián 20014, Spain

$\dagger$ Electronic supplementary information (ESI) available. See DOI: 10.1039/ d0ma00370k
}

different applications as adhesives, additives in thermoplastics (to increase flexibility and stability), and matrices for composite materials used in aerospace, structural, and sport applications. ${ }^{3-5}$ ESO is known as a plasticizer or stabilizer of thermoplastics such as poly(vinyl chloride). ${ }^{6}$ The mechanical behaviour and chemical resistance of EVO-based thermosets are strongly dependent on the nature of the crosslinking reactions, ranging from the chemical aspects of the hardener to physical ones such as the process parameters. There is scarce information regarding EVO curing with dicarboxylic acids. ${ }^{7-13}$ However, an important aspect of the epoxy/acid reaction is the formation of $\beta$-hydroxyesters that are responsible for transesterification reactions, which allow the recyclability of the polymeric networks. ${ }^{14,15}$ Another reaction favouring network chain reorganization is the metathesis of disulfide covalent bonds by a dynamic exchange in the main or side chains, usually triggered by external stimuli, such as pressure or temperature, known as the disulfide exchange reaction. ${ }^{16,17}$ Aromatic disulfide bridges are relatively often introduced into polymeric networks such as polyurethanes, ${ }^{18}$ poly(urea-urethanes) ${ }^{19}$ and epoxy resins. ${ }^{20}$

In this work, we studied the influence of the epoxy/hardener ratio as a key parameter influencing the reprocessing ability of the thermosets. Two kinds of EVOs were analysed and compared: ELO, ESO and their mixture (ELO + ESO). 2,2'-Dithiodibenzoic acid (DTBA) was used as a dynamic hardener. Our team already studied how the initiators influence the copolymerization of ELO/DTBA, ${ }^{13,21}$ but, to the best of our knowledge, this study reports for the first time the effect of the ratio of the thermoset systems on the chemical recyclability 
and the thermal reprocessing properties. The epoxy/acid reactions were monitored via differential scanning calorimetry (DSC) and infrared spectroscopy (FT-IR) analyses. The obtained thermosets and the reprocessed resins were analysed and characterized by using thermogravimetric analysis (TGA) and dynamic mechanical analysis (DMA). Finally, the swelling properties were exploited to chemically recycle the thermosets. In contrast to Leibler et al., ${ }^{22}$ who cured ENR (Epoxidized Natural Rubber) with dithiodibutyric acid and performed swelling in THF, we show here the ability of the formed thermosets to swell until dissociation in THF. The obtained material was further filtered from THF, dried and mechanically reprocessed, and its properties were compared with the virgin thermoset. The thermo-mechanical properties were evaluated to understand how the structural ratio between the comonomers influenced the chemical and mechanical recycling and moreover affected the material's performance.

\section{Results and discussion}

\section{Influence of the EVO/DTBA ratio on the crosslinking by DSC investigation}

To evaluate the effect of $R$ ratio on the crosslinking reaction and also the effect of mixing ELO and ESO monomers on the reaction reactivity, the prepared formulations (displayed in Table S2, ESI $\dagger$ ) were studied by dynamic DSC analysis.

The DSC thermograms obtained during the heating of the formulations for curing are presented in Fig. 1 and Fig. S1 (ESI $\dagger$ ), where the exothermic events were associated with the crosslinking reactions. The formulation system with the ratio $R=1$ displays the highest reaction enthalpy (Table 1 ) in accord with the results of Williams et al. ${ }^{14}$ on ESO/citric acid (CA) curing, or with Leibler et al. ${ }^{15}$ on DGEBA cured with a mixture of fatty dicarboxylic and tricarboxylic acids. Ma et $a .^{23}$ selected the equivalent ratio of $1: 1$ for curing epoxidized sucrose soyate

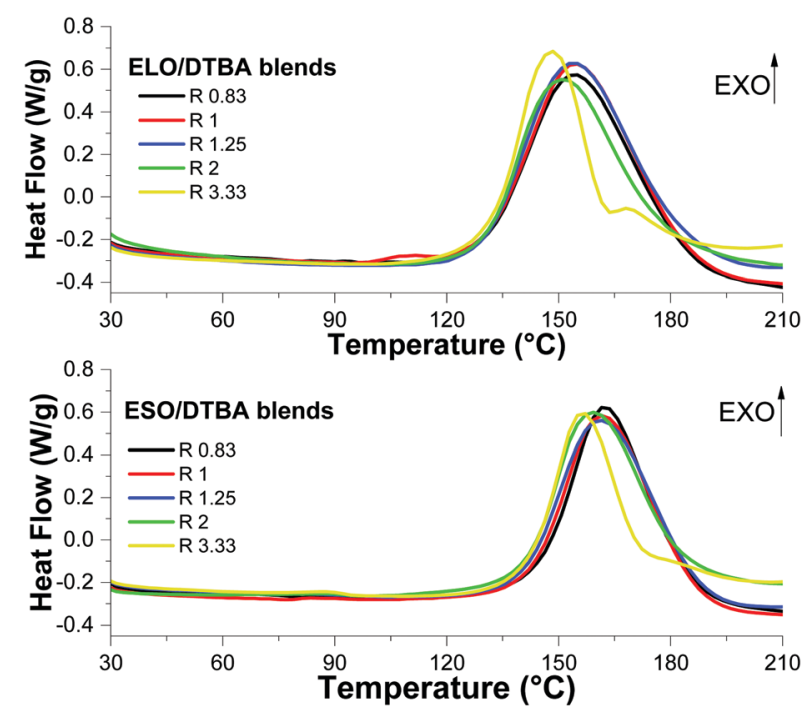

Fig. 1 DSC thermograms of the curing process at $10{ }^{\circ} \mathrm{C} \mathrm{min}$ m $^{-1}$ of ELO/ DTBA (A) and ESO/DTBA (B) formulations.
Table 1 DSC results on the crosslinking reaction studies of selected systems

\begin{tabular}{|c|c|c|c|c|}
\hline Formulations & Ratio e/a & $\Delta H\left(\mathrm{~J} \mathrm{~g}^{-1}\right)$ & $T_{\text {peak }}\left({ }^{\circ} \mathrm{C}\right)$ & Reaction interval \\
\hline ELO/DTBA-0.83 & $1: 1.2$ & $182 \pm 3$ & $151 \pm 1$ & $130-185$ \\
\hline ELO/DTBA-1 & $1: 1$ & $197 \pm 3$ & $151 \pm 1$ & $130-184$ \\
\hline ELO/DTBA-1.25 & $1: 0.8$ & $184 \pm 3$ & $151 \pm 1$ & $131-180$ \\
\hline ELO/DTBA-2 & $1: 0.5$ & $158 \pm 3$ & $148 \pm 1$ & $131-180$ \\
\hline ELO/DTBA-3.33 & $1: 0.3$ & $125 \pm 3$ & $146 \pm 1$ & $132-164$ \\
\hline ESO/DTBA- 0.83 & $1: 1.2$ & $139 \pm 3$ & $160 \pm 1$ & $144-186$ \\
\hline ESO/DTBA-1 & $1: 1$ & $149 \pm 3$ & $159 \pm 1$ & $142-187$ \\
\hline ESO/DTBA-1.25 & $1: 0.8$ & $146 \pm 3$ & $158 \pm 1$ & $162-188$ \\
\hline ESO/DTBA-2 & $1: 0.5$ & $135 \pm 3$ & $156 \pm 1$ & $146-184$ \\
\hline ESO/DTBA-3.33 & $1: 0.3$ & $106 \pm 3$ & $154 \pm 1$ & $157-173$ \\
\hline ELO/ESO/DTBA-0.83 & $1: 1.2$ & $156 \pm 3$ & $159 \pm 1$ & $132-192$ \\
\hline ELO/ESO/DTBA-1 & $1: 1$ & $174 \pm 3$ & $155 \pm 1$ & $134-185$ \\
\hline
\end{tabular}

(ESS) with several natural acids: citric acid, D,L-malic acid, L-tartaric acid, malonic acid, oxalic acid, and glutaric acid.

The ELO/DTBA-1 system was confirmed as the more reactive with a reaction enthalpy of $197 \mathrm{~J} \mathrm{~g}^{-1}$, followed by the system ELO/ESO/DTBA-1 with an enthalpy of $174 \mathrm{~J} \mathrm{~g}^{-1}$ while the formulation ESO/DTBA-1 gave a reaction enthalpy of $146 \mathrm{~J} \mathrm{~g}^{-1}$. This result can be linked to the higher epoxy content in ELO, 5.61 meq. $\mathrm{g}^{-1}$, compared to ESO that has 4.66 meq. $\mathrm{g}^{-1}$ epoxy functions. This can explain why ELO/ESO blends show a higher reaction enthalpy compared to the ESO based system. The enthalpies and interval of reactions for the ELO + ESO mixture display comparable trends with those of ELO based systems.

Upon increasing the percentage of acids in the curing mixtures with ELO, a decrease of reaction enthalpy from 197 to $184 \mathrm{~J} \mathrm{~g}^{-1}$ was measured (Table 1). Comparable results were obtained for the ESO based systems, with enthalpies decreasing from 149 to $135 \mathrm{~J} \mathrm{~g}^{-1}$, while for the ELO/ESO systems, the reaction enthalpies varied from 174 to $156 \mathrm{~J} \mathrm{~g}^{-1}$. Matharu et al. ${ }^{8}$ studied an excess of epoxy functionality ( $R$ of 0.7 for acid/epoxy groups) for ELO crosslinked by different DCAs in the presence of DMAP. The authors reported the lowest reactivity when ELO was cured with aliphatic dicarboxylic acids with a high number of carbon atoms $-\mathrm{C}_{36}\left(173 \mathrm{~J} \mathrm{~g}^{-1}\right)$, and, in contrast, the reaction enthalpy increased up to $261 \mathrm{~J} \mathrm{~g}^{-1}$ in the presence of $\alpha, \omega$-DCA (from $\mathrm{C}_{6}$ to $\mathrm{C}_{18}$ ).

From Fig. 1 and Table 1, we can notice a reduction of reaction temperature interval for the systems with a large excess of epoxy monomer in the mixtures. These results are in contrast with those of Zeng et al., ${ }^{9}$ who reported for ESO/DCA curing (adipic acid, sebacic acid and 1,2-dodecanedicarboxylic acid), an optimal ratio $R=0.7$. The authors attributed the decrease of reaction enthalpy for $R<0.7$ to an insufficient amount of acids in the blend. In our systems, the decrease of reaction enthalpy for $R<1$ can be related to the fact that the systems are out of functional epoxy-acid copolymerization stoichiometry. For the systems with $R>2$, secondary reactions (homopolymerizations, etherifications) start to occur at high temperatures, as we can observe in the DSC thermograms by the presence of small exotherms at around 170 and $180{ }^{\circ} \mathrm{C}$.

\section{Influence of the epoxy/acid ratio analyzed by FT-IR studies}

EVO/DTBA mixtures and thermosets were prepared according to the protocol presented in Experimental Section. Due to the 

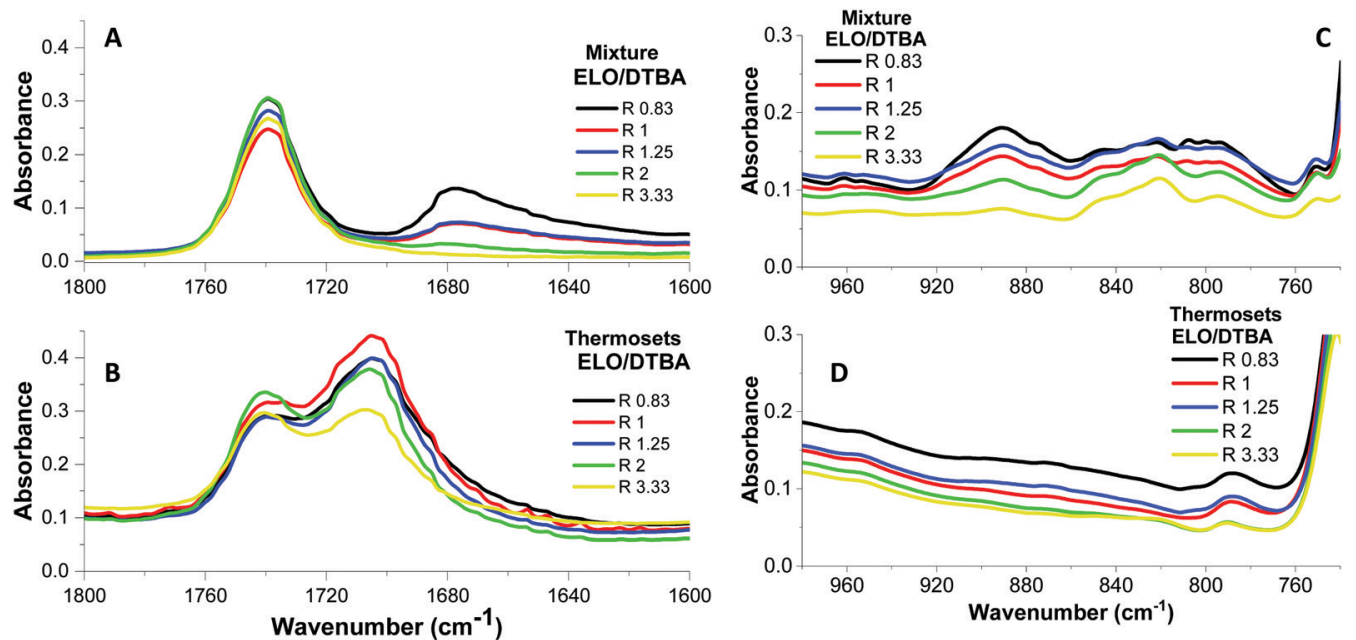

Fig. 2 FTIR spectra for ELO/DTBA systems: ester + acid regions in the starting mixtures (A) and ester + new carbonyl regions in the cured resins (B); epoxy $920-780 \mathrm{~cm}^{-1}$ regions in the initial mixtures (C) and in the final thermosets (D).

insufficient hardener amount, it was not possible to obtain cured thermosetting resins for the systems of ESO/DTBA $R=2$ and $R=3.33$ and for ELO/DTBA $R=3.33$. At the same time, due to the low epoxy content in ESO, it was not possible to crosslink the system of ESO/DTBA $R=2$.

Fig. 2 illustrates the FTIR spectral comparison of reactive mixtures and those of the corresponding thermosets for ELO/ DTBA systems at selected ratios. Fig. 2A and B presents the region from 1800 to $1600 \mathrm{~cm}^{-1}$. In this region, we can observe the presence of reference peaks in Fig. $2 \mathrm{~A}$, i.e., $1 /$ that of $\mathrm{C}=\mathrm{O}$ from DTBA stretching at $1680 \mathrm{~cm}^{-1}$, a peak that decreases continuously in intensity in the systems with an excess of ELO, and 2/that at $1740 \mathrm{~cm}^{-1}$, the stretching of $\mathrm{C}=\mathrm{O}$ of ELO triglyceride. As we can see in Fig. 2B, after the curing, the band at $1680 \mathrm{~cm}^{-1}$ completely disappears while a new peak emerged at $\sim 1700 \mathrm{~cm}^{-1}$, confirming the formation of an ester by epoxy/acid polyaddition esterification reactions. From this figure, we can notice that the intensity of this new ester peak is smaller in the systems with an excess of epoxy. For the system $R=3.33$, the two esters: that of ELO's triglyceride, and that of the new formed esters have the same intensity. The characteristic absorption of the epoxy groups can be observed in Fig. 2C, together with the homopolymerizations in the systems with an excess of epoxy.

Even if the ELO/DTBA-3.33 system did not undergo satisfactory hardening, the epoxy groups completely disappeared in the spectrum, indicating that the ring opening reactions occurred via homopolymerization instead of copolymerization. This FTIR study highlights that the ratio $R=1$ is optimal considering the higher copolymerization conversion percentage of epoxy, acid and ester functions, for all the studied systems (Table 2).

Table 2 Copolymerization conversion \% for EVO/DTBA systems

\begin{tabular}{|c|c|c|c|}
\hline Formulation & ELO/DTBA & ESO/DTBA & $\begin{array}{l}\mathrm{ESO} / \mathrm{ELO} / \\
\mathrm{DTBA}\end{array}$ \\
\hline
\end{tabular}

\begin{tabular}{lllllllllllll}
\hline Ratio & 0.83 & 1 & 1.25 & 2 & 3.33 & 0.85 & 1 & 1.25 & 2 & 3.33 & 0.83 & 1
\end{tabular}

$\begin{array}{lllllllllllll}\text { Conversion } \% & 84 & 99 & 96 & 74 & 59 & 79 & 88 & 81 & 65 & 44 & 81 & 92\end{array}$
From this study, we can notice that an excess of epoxy functions leads to a higher conversion than that of a system with an excess of DTBA hardener. For example, for the ELO/DTBA-1.25 system, the conversion is around $96 \%$ versus $84 \%$ for ELO/ DTBA-0.83. The copolymerization conversion decreases drastically from $R=1$ to $R=3.33$, confirming that secondary reactions took place in out-of-stoichiometry systems.

For the ESO/DTBA (Fig. S3A, ESI $\dagger$ ) and ELO/ESO/DTBA systems, the results show the same trend as that of the discussed systems with ELO.

\section{Thermo-mechanical analysis of virgin and recycled thermosets}

The properties of the virgin and reprocessed materials were studied, in order to highlight firstly how the ratio $R$ influences the recyclability and secondly to evaluate how the recycling affects the thermomechanical properties.

Table 3 and Table S3 (ESI $\dagger$ ) report the reprocessing conditions for the analysed systems. A fixed reprocessing temperature of $160{ }^{\circ} \mathrm{C}$ under 60 bars of pressure was applied to all the materials. Table 3 shows that for $R>1$, the reprocessing duration decreases, from 30 to 10 and 5 minutes, and the recycled materials also changed in their properties, becoming more elastic and transparent for $R=2$. This result can be connected to the fact that the epoxy groups that are in excess promote homopolymerization and/or etherification, therefore the as created chains are more flexible compared to those formed by copolymerization with an aromatic hardener. However, with a lack of hardener, the crosslinking density is low, therefore the chains have more freedom to rearrange. The elasticity and transparency diminish with the hardener percentage due to a higher crosslink density in the copolymer network.

ESO recycled thermosets, in contrast with ELO resins, have a higher elasticity at $R<1$ as well, due to a lower epoxy content in ESO, guaranteeing high flexibility in the thermosetting resins and consequently in the reprocessed materials. Likewise, the mixture of ELO and ESO produced rigid, hard or elastic materials following the behaviour of the ELO based thermosets. 
Table 3 Appearance of samples after reprocessing at $160{ }^{\circ} \mathrm{C}$ and 60 bars of ELO/DTBA thermosets. The durations of the process are indicated

\begin{tabular}{lllll}
\hline Ratio & 0.83 & 1 & 1.25 & 2 \\
\hline ELO/DTBA M*-reprocessed resins & 30 & 30 & 10 & 5
\end{tabular}

\section{Glass transition study}

The virgin and reprocessed thermosets were analysed using DSC for glass transition $\left(T_{\mathrm{g}}\right)$ evaluation, and the results are displayed in Fig. 3A and summarized in Table 4. ELO/DTBA thermosets at different $R$ ratios show the largest differences in $T_{\mathrm{g}}$ values, from 79 to $53{ }^{\circ} \mathrm{C}$, i.e. from the system in excess of epoxy to that in excess of hardener. It is interesting to notice that both systems at ratios $R=1$ and $R=1.25$ are characterized by the same value of $T_{\mathrm{g}}$, around $76{ }^{\circ} \mathrm{C}$. Boquillon et al. ${ }^{24}$ reported that the $T_{\mathrm{g}}$ values remain constant in the epoxy/acid ratio range from 1.66 to 1 in the thermoset resins of ELO cured with THPA (cis-1,2,3,6-tetrahydrophthalic anhydride) in the
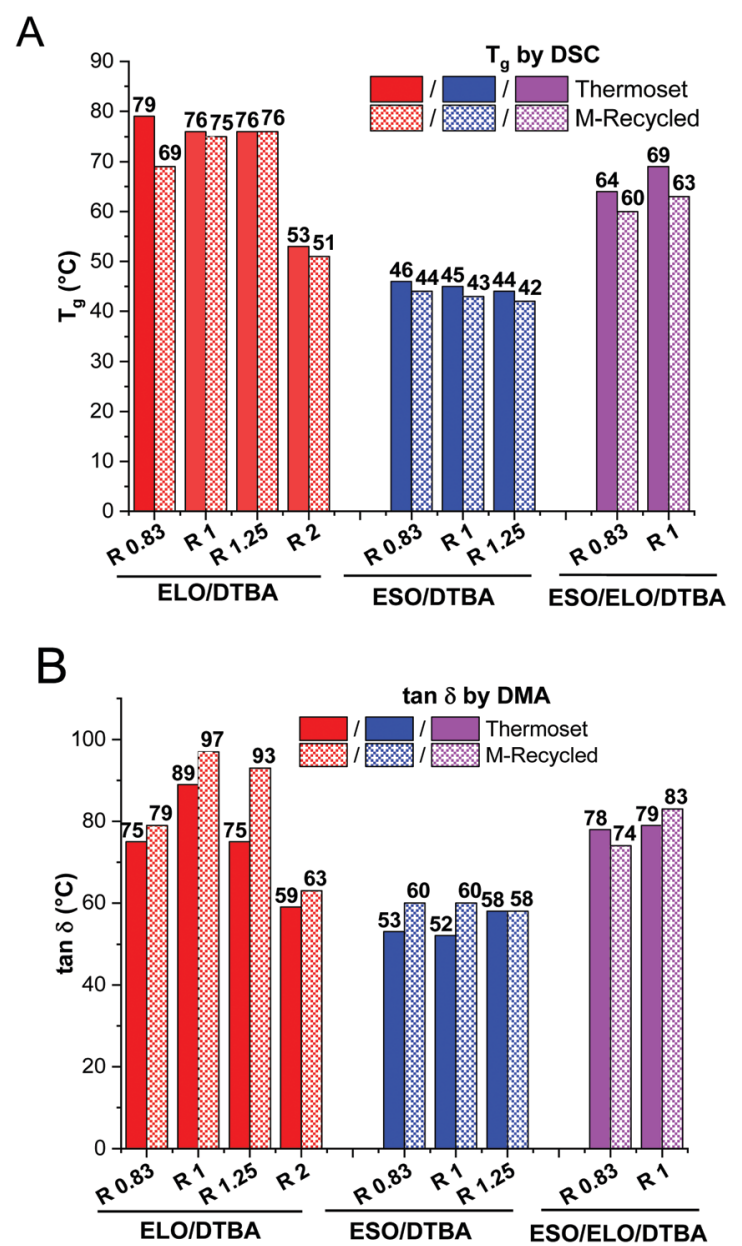

Fig. 3 Comparison of virgin and mechanically reprocessed materials. Glass transition values evaluated by dynamic DSC analysis (A) and $\tan \delta$ measured by DMA (B).
Table 4 Glass transition values determined by DSC for virgin and reprocessed thermosets

\begin{tabular}{lccccccccc}
\hline & \multicolumn{1}{c}{ ELO/DTBA } & \multicolumn{4}{c}{ ESO/DTBA } & \multicolumn{2}{c}{ ESO/ELO/DTBA } \\
\hline$T_{\mathrm{g} \text { of virgin }}$ & 79 & 76 & 76 & 53 & 46 & 45 & 44 & 64 & 69 \\
$T_{\mathrm{g}}$ of $\mathbf{M}^{*}$-recycled & 69 & 75 & 76 & 51 & 44 & 43 & 42 & 60 & 63
\end{tabular}

presence of 2-methylimidazole. Wingard et $a .^{25}$ analysed different ratios of DGEBA/methylene dianiline (MDA) and correlated the higher $T_{\mathrm{g}}$ value obtained in the case of the stoichiometric ratio with a restricted molecular mobility in correlation with the highest degree of crosslinking.

Galy et $a .^{26}$ by a thorough study of the crosslinking of DGEBA with several diamines reported decreasing $T_{\mathrm{g}}$ values in the systems with an excess of epoxy, explained by the presence of unreacted epoxy functions as mobile pendant chain ends. As presented in Table 4, the ESO/DTBA thermosets are characterized by very close values of $T_{\mathrm{g}}$, from 46 to $44{ }^{\circ} \mathrm{C}$, for $R=0.83$ and 1,25 , respectively. The mixture of (ELO + ESO) systems have intermediate $T_{\mathrm{g}}$ values, ranging from 64 to $69{ }^{\circ} \mathrm{C}$ for ELO/ESO/DTBA with $R=0.83$ and $R=1$, respectively. Therefore, these thermosets are characterized by $T_{\mathrm{g}}$ values closer to those of the ELO based thermosets.

After the mechanical reprocessing, a decrease of the $T_{\mathrm{g}}$ to some degree can be observed for ELO/DTBA off-stoichiometric systems with $R=0.83$ and 2 , being more pronounced for the system with an excess of hardener. The other systems have a constant value of $T_{\mathrm{g}}$ before and after the reprocessing.

\section{DMA analysis}

Fig. 4 illustrates the DMA results in the form of $\tan \delta=f(T)$ obtained during the thermomechanical analysis of virgin and reprocessed thermosets. Overall, for all ratios, it is possible to observe that all materials, virgin or recycled, exhibit a single $\tan \delta$ transition (Table 5 and Tables S4, S5, ESI $\dagger$ ). Furthermore, we can also note that these curves are symmetric. These results show that the networks are structurally homogeneous, characterized by the same nature of the chains. This result is very important concerning the impact of the reprocessing on material structure and morphology.

Moreover, the obtained $\tan \delta$ values are in good correlation with that of $T_{\mathrm{g}}$ measured by DSC, with just a small difference. For example, the maximum value of $\tan \delta$ was obtained for $R=1$ and not for 0.83 as with the $T_{\mathrm{g}} \mathrm{s}$. The obtained results are in line with those of Boquillon et al., ${ }^{24}$ who reported that for $R<1$, a decrease of $\tan \delta$ was observed, this result being correlated with partly reacted hardener units that can freely move and increase the interchain distance. 

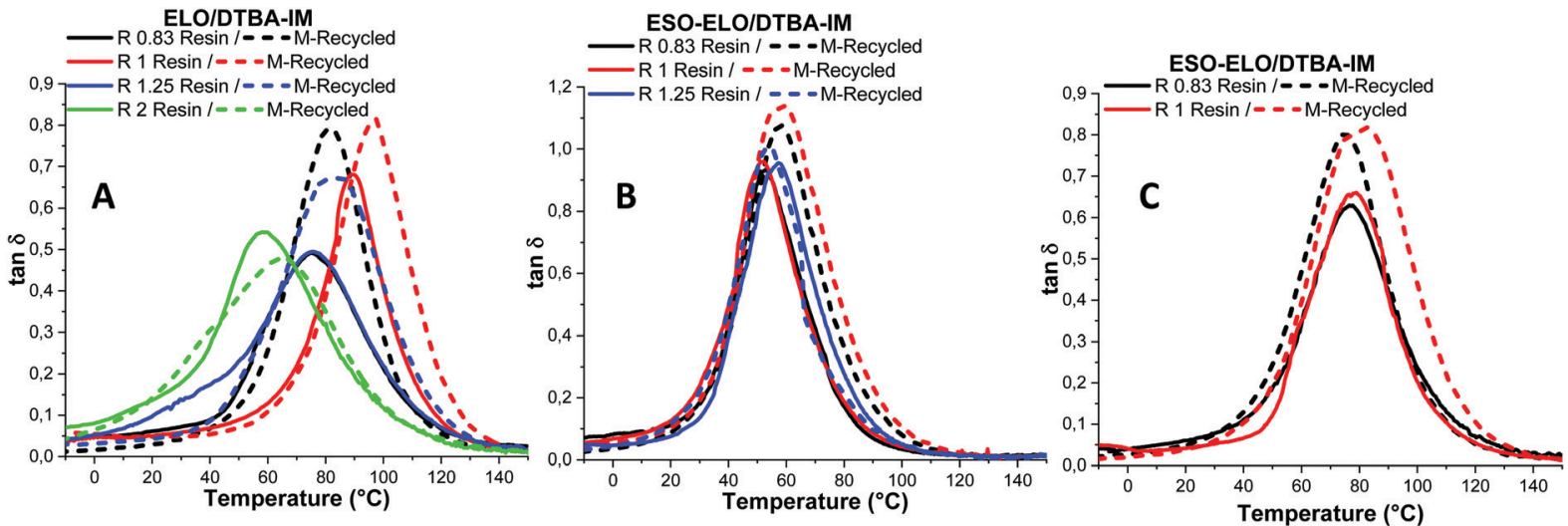

Fig. 4 DMA results: $\tan \delta$ vs. temperature analysis for virgin and recycled materials with the composition: (A) ELO/DTBA, (B) ESO/DTBA and (C) ELO-ESO/DTBA.

The mechanically reprocessed resins exhibit a comparable $\tan \delta$ trend to that of the virgin ones with a shift to higher damping factor transitions (Fig. 4B). This increasing of the $\tan \delta$ amplitude observed in the reprocessed samples is proof of a decrease in the crosslinking density. This means that during the reprocessing, the chemical dynamic bonds rearranged in a different manner inside the network. However, the systems with an excess of epoxy seem to be less impacted by the reprocessing. Indeed, for the ELO/DTBA-2 system, the $(\tan \delta)_{\max }$ values before and after the recycling process are very close: 0.48 and 0.54 .

Fig. S4 (ESI $\dagger$ ) illustrates the dependence of the storage modulus $E^{\prime}$ on temperature. The thermoset systems with $R>1$ exhibit a low modulus in the rubbery plateau region, especially the ELO based systems. The area above the $\alpha$ relaxation and the value of the modulus in that area depend on many factors, such as the chemical structure, the ratio of the matrix components and the crosslink density of the network. The thermosets with $R=0.83$ exhibit a higher $E^{\prime}$ in the rubbery plateau. This result corroborates with that reported by Curtis $e t ~ a l .{ }^{27}$ for epoxidized canola oil cured with phthalic anhydride (PA) thermoset in an equimolar ratio and with an excess of hardener. The authors explained that the higher $E^{\prime}$ modulus after the $T_{\mathrm{g}} \mathrm{s}$ for the equimolar ratio can be associated with the presence of side chains that are able to move. An increase of hardener led to a greater cross-link density, so fewer chains were available for relaxation and consequently the modulus was lower.

The calculations made using eqn (3) allowed us to evaluate the crosslink densities of the virgin and mechanically reprocessed materials in the rubbery plateau. These results are summarized in Table 5 that displays the comparison of the crosslink densities for ELO/DTBA thermoset systems. The system with $R=1$ has the higher crosslink density, of around $1.25 \mathrm{mmol} \mathrm{cm}{ }^{-3}$.

This value decreases in the system with an excess of DTBA $(R=0.83)$ at $1.19 \mathrm{mmol} \mathrm{cm}^{-3}$. For the systems with an excess of epoxide, $R=1.25$ and 2 , the crosslink density decreases to 0.61 and $0.28 \mathrm{mmol} \mathrm{cm}{ }^{-3}$. These results are in agreement with the data reported by Williams et al. ${ }^{14}$ for DGEBA/citric acid thermosets, which presented a sensible decrease of the crosslink density when the ratio $R$ increases from 1 to 2 .

Even if the $E^{\prime}$ values in the rubbery plateau are increased after the reprocessing, the crosslink density diminishes, except the system with $R=2$ where the crosslink density augments after reprocessing from 0.28 to $0.59 \mathrm{mmol} \mathrm{cm}^{-3}$. In our previous work, ${ }^{21}$ we explained that this increase of the crosslink density can be attributed to the dual mechanism of the network's internal rearrangement occurring during reprocessing. ESO/DTBA systems revealed a similar trend to that of the ELO based systems (Table S4, ESI $\dagger$ ), with lower crosslink

Table 5 Thermomechanical properties of the virgin and reprocessed resins with different ratios

\begin{tabular}{|c|c|c|c|c|c|c|}
\hline ELO/DTBA & & $\tan \delta\left({ }^{\circ} \mathrm{C}\right)$ & $(\tan \delta)_{\max }$ & $E^{\prime}$ glassy plateau (MPa) & $E^{\prime}$ rubbery plateau $(\mathrm{MPa})$ & Crosslink density $\left(\mathrm{mmol} \mathrm{cm}^{-3}\right)$ \\
\hline \multirow[t]{3}{*}{$R=0.83$} & $\mathrm{~V}^{*}$ & 75 & 0.49 & 2000 & 4.48 & 1.19 \\
\hline & $\mathrm{M}-\mathrm{R}^{*}$ & 79 & 0.77 & 2000 & 9.27 & 0.87 \\
\hline & $\mathrm{C}-\mathrm{M}-\mathrm{R}^{*}$ & 72 & 0.58 & 2000 & 8.37 & 0.79 \\
\hline \multirow[t]{3}{*}{$R=1$} & $\mathrm{~V}^{*}$ & 89 & 0.68 & 1560 & 8.60 & 1.25 \\
\hline & $\mathrm{M}-\mathrm{R}^{*}$ & 97 & 0.82 & 1720 & 8.90 & 0.81 \\
\hline & $\mathrm{C}-\mathrm{M}-\mathrm{R}^{*}$ & 78 & 0.56 & 1730 & 8.73 & 0.86 \\
\hline \multirow[t]{3}{*}{$R=1.25$} & $\mathrm{~V}^{*}$ & 75 & 0.49 & 2800 & 4.42 & 0.61 \\
\hline & $\mathrm{M}-\mathrm{R}^{*}$ & 93 & 0.60 & 1360 & 5.79 & 0.52 \\
\hline & $\mathrm{C}-\mathrm{M}-\mathrm{R}^{*}$ & 90 & 0.69 & 2240 & 10.59 & 0.99 \\
\hline \multirow[t]{3}{*}{$R=2$} & $\mathrm{~V}^{*}$ & 59 & 0.54 & 1450 & 2.99 & 0.28 \\
\hline & $\mathrm{M}-\mathrm{R}^{*}$ & 63 & 0.48 & 1900 & 6.54 & 0.59 \\
\hline & $\mathrm{C}-\mathrm{M}-\mathrm{R}^{*}$ & 65 & 0.53 & 2000 & 7.78 & 0.78 \\
\hline
\end{tabular}

$\mathrm{V}^{*}$ virgin, $\mathrm{M}-\mathrm{R}^{*}$ mechanically reprocessed, $\mathrm{C}-\mathrm{M}^{*}$ chemically and mechanically recycled. 


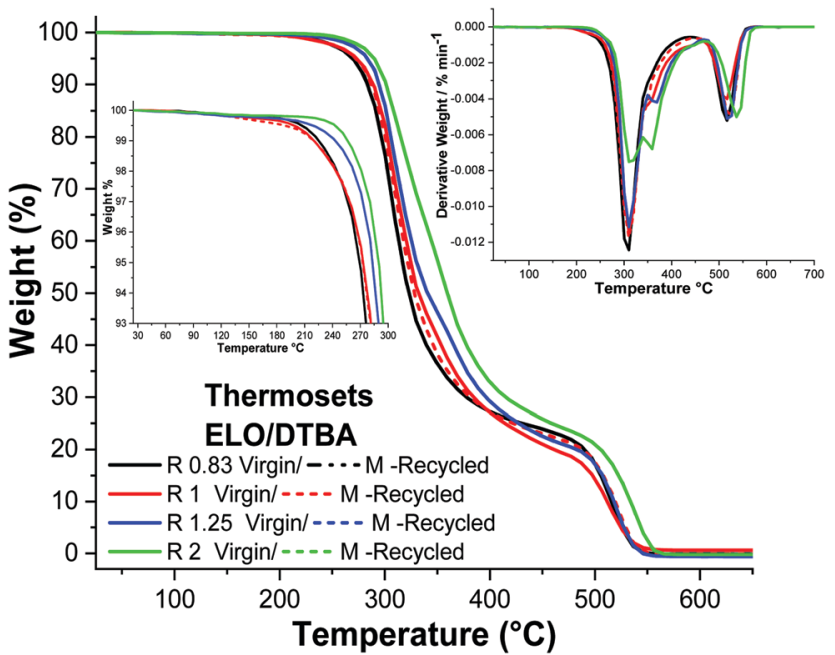

Fig. 5 TGA, DTG and zoomed in results of the region from 25 to $300{ }^{\circ} \mathrm{C}$ for ELO/DTBA systems during heating at $10{ }^{\circ} \mathrm{C} \mathrm{min}-1$ under air.

densities in systems with an excess of epoxide. But, ESO/DTBA $R=1$ has the lowest network density compared with the other ratios, while after reprocessing, the value increases from 0.082 to $0.31 \mathrm{mmol} \mathrm{cm}^{-3}$. Finally, the ELO/ESO/DTBA systems have intermediate properties (Table S4, ESI $\dagger$ ), and again the ELO/ ESO/DTBA $R=1$ thermoset has the higher crosslinking density.

\section{Thermal stability analysis}

The thermal stabilities were tested to detect how the ratio $R$ or the recycling process affects the material stability. The TGA results obtained for the ELO/DTBA systems are shown in Fig. 5, displaying that the $T_{5 \%}$ values range from $271{ }^{\circ} \mathrm{C}$ for $R=0.83$ to $291{ }^{\circ} \mathrm{C}$ for $R=2$. The data concerning ESO/DTBA and those of the ELO/ESO/ DTBA systems are given in Fig. S5 and S6 (ESI $\dagger$ ). The results show that the reprocessing did not affect the thermal stability, with thermograms sometimes completely superposed between virgin and reprocessed materials (Fig. 5 and Table S6, ESI $\dagger$ ).

The lower thermal stability of the system with an excess of DTBA can be associated with the degradation of the hardener $\left(T_{5 \% \text {, DTBA }}=250{ }^{\circ} \mathrm{C}\right)$. In contrast, the systems with $R>1$ have a higher thermal stability, probably an effect of the secondary reactions involving the excess of the epoxide, such as homopolymerization or etherification. Comparable results were reported previously for ELO/diacid hardener (dipropylene glycol dimaleate) thermosets: the thermal stability ranged from $T_{10 \%} 329{ }^{\circ} \mathrm{C}$ for $R=1.25$ to $315{ }^{\circ} \mathrm{C}$ for $R=1 .^{12}$

The DTG curves clearly show that the materials exhibit a two-stage thermal decomposition under an oxidative atmosphere (air). The first step is mainly assigned to the pyrolysis of the chains and the second step is assigned to the thermooxidative degradation of the products formed in the first step, occurring around $450{ }^{\circ} \mathrm{C}$.

Chemical recycling and characterization of recycled materials

According to Leibler et al., ${ }^{15,22}$ vitrimers are crosslinked materials able to swell in adequate solvents, the swelling experiment

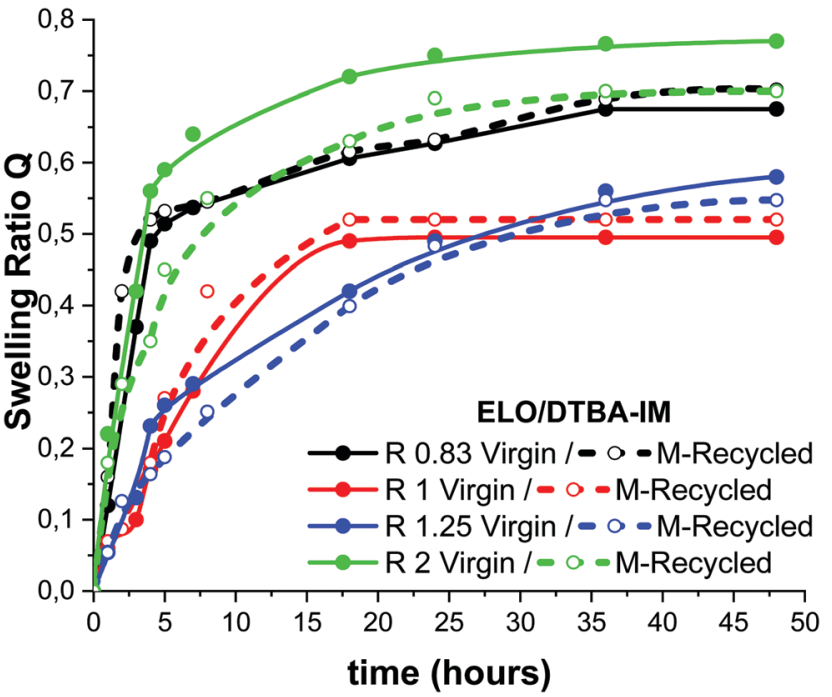

Fig. 6 Swelling behaviour in toluene of the virgin and recycled ELO/DTBA thermosets.

giving information concerning the network. To study the swelling behaviour of the virgin and recycled thermosets, all the selected ratio samples were immersed in toluene at room temperature for 48 hours. Thereafter, the swelling ratio $Q$ was calculated according to eqn (4) and plotted as a function of time in Fig. 6 and Fig. S7 (ESI $\dagger$ ). The systems with $R=1$ show the least swelling together with the ratio of 1.25 that exhibits comparable behaviour. Among all the virgin systems, that with a large excess of epoxy, $R=2$, shows the highest swelling, in good agreement with the previous results related to the lowest crosslinking density. The same trend can be observed for the system with an excess of hardener, $R=0.83$. Fig. 6 shows that after $35 \mathrm{~h}$, the swelling ratio reaches a plateau for most of the systems.

The reprocessed resins show very similar swelling properties to those of the virgin ones. An exception to this behaviour occurs in the systems with $R>1$ that exhibit less swelling, in agreement with the increased crosslink densities and mechanical properties, compared to virgin samples, as evaluated by DMA analysis. For example, the reprocessed $R=2$ resin exhibits a decrease of the swelling ratio compared with the virgin material, a result corroborating with the three times higher crosslink density measured for the reprocessed material compared with the virgin one, as determined by DMA. Similar results were presented by Leibler et al. ${ }^{22}$ for epoxidized natural rubber (ENR) cured by dithiodibutyric acid (DTDB), a disulphide diacid hardener.

According to the definition of vitrimers, ${ }^{15,28}$ these materials do not exhibit a loss in structural integrity, even at elevated temperatures, ${ }^{29}$ in common solvents. For these reasons, we studied the solvent stability of the prepared virgin samples in ethanol, methanol, acetone, water, DMF, DCM, DMSO and THF, at room temperature, for 48 hours. The solvent stability tests are displayed in Fig. S8 (ESI $\dagger$ ). All the thermosets exhibited good stability in ethanol, methanol, acetone and water. Swelling in toluene and dichloromethane was observed for all the resins and a dissociative behaviour was observed in THF, DMSO and DMF.

At the end of the experiment in acetone, ESO/DTBA thermosets somewhat disintegrated into the solvent. Thereafter, the 


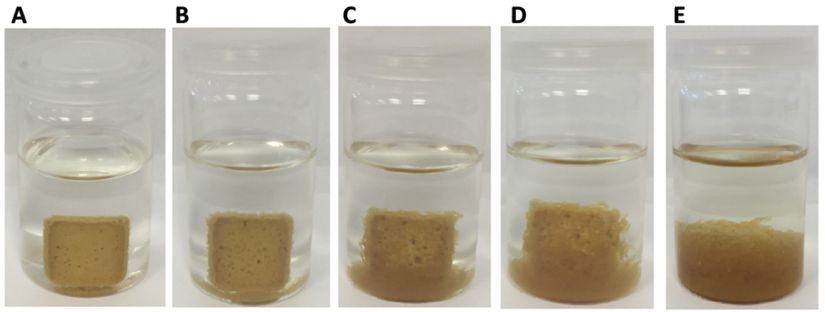

Fig. 7 Evolution in time of ELO/DTBA $R=0.83$ thermoset immersed in THF at room temperature: initial time (A), 10 minutes (B), 20 minutes (C), 30 minutes (D) and 60 minutes (E).

solid part was dried and the soluble percentage of the resin was determined (Table S7, ESI $\dagger$ ). In all cases, only a very small fraction $(<1 \%)$ of resin was released into the solvents, possibly due to unreacted molecules from out-of-stoichiometry systems.

A different result was obtained in the experiments using THF. Fig. 7 shows the evolution of the immersed samples in THF. After 10 minutes, the samples start to disintegrate, and after 60 minutes, they completely separated into small fragments. Then, these fragmented parts were dried in an oven at $80{ }^{\circ} \mathrm{C}$ for 24 hours then weighed and analyzed. Table S7 (ESI $\dagger$ ) highlights that no mass loss occurred. At the end of the experiment, the sample loss was $<0.2 \%$.

To study the chemical stability of the thermosets in THF, the recuperated samples were dried and analysed using FTIR spectroscopy. Fig. S8 (ESI $\dagger$ ) shows the FT-IR spectra of ELO/DTBA $R=0.83$ thermosets. By comparing the FTIR spectra of the initial thermoset and that immersed in THF, it is clear that no changes occurred, the spectra are identical. Moreover, the dried samples were mechanically reprocessed using the same protocol applied to the virgin thermosets $\left(160{ }^{\circ} \mathrm{C}\right.$ and 60 bars) and, surprisingly, the systems with $R<1$ required a shorter time (10 minutes compared with 30 minutes) to reprocess satisfactorily.

Fig. 8 shows the appearance of ELO/DTBA $R=0.83$ thermosets after reprocessing and after chemical recycling in THF followed by reprocessing.

The last one, associated with the chemical-mechanical recycling (C-M), displays a higher flexibility and transparency. This was already observed after the reprocessing of the systems with $R=2$, without chemical recycling in THF. The C-M recycled materials were studied by thermo-mechanical analysis to evaluate how the two combined recycling processes affected the material's properties and to compare them with those only mechanically reprocessed.

Fig. 9 and Table S8 (ESI $\dagger$ ) report the results of TGA analysis of ELO/DTBA systems mechanically and C-M recycled. A comparison of the obtained results shows that the systems with
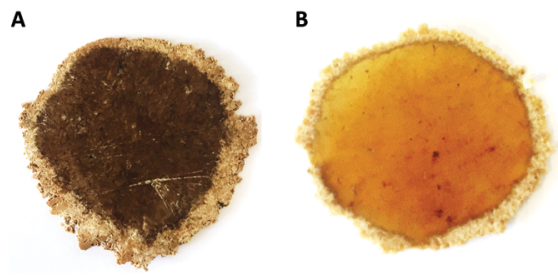

Fig. 8 Appearance of ELO/DTBA $R=0.83$ thermosets after mechanical (M) (A) and chemical-mechanical (C-M) (B) recycling and reprocessing.

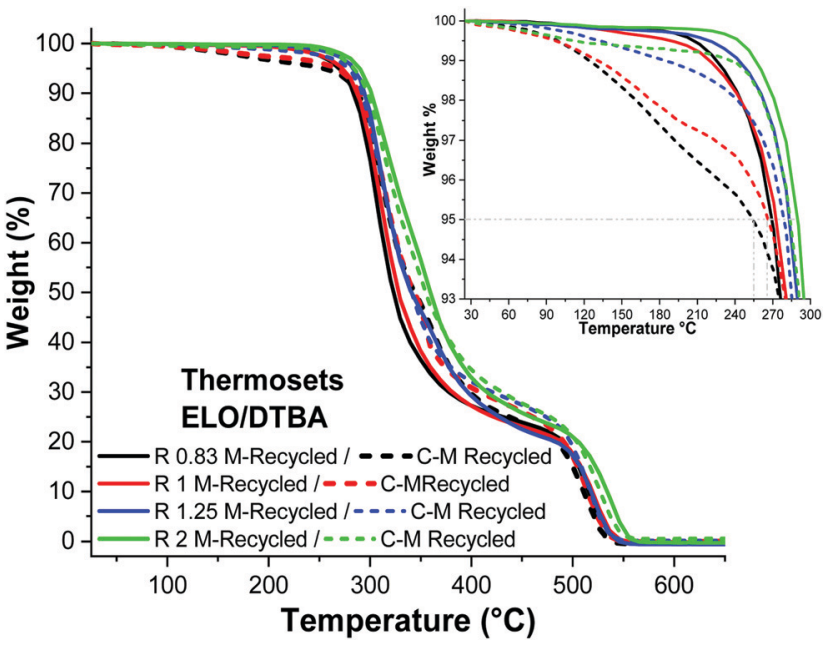

Fig. 9 TGA and zoomed in results of ELO/DTBA materials after mechanical or $\mathrm{C}-\mathrm{M}$ reprocessing.

$R \leq 1$ are the most affected by the $\mathrm{C}-\mathrm{M}$ reprocessing with a decrease of $T_{5 \%}$ from 273 to $250{ }^{\circ} \mathrm{C}(R=0.83)$.

For the other systems, the TGA results show that the $T_{\text {onset }}$ decreased negligibly, and all C-M reprocessed materials exhibited a similar thermal stability to that of the mechanically reprocessed materials.

Fig. 10 illustrates the DMA results comparing ELO/DTBA virgin materials, those mechanically reprocessed (M) and those chemically-mechanically reprocessed (C-M). The C-M reprocessed samples with $R \leq 1$ exhibit a decrease of the damping factor, compared with the mechanically reprocessed materials. Different behaviour can be observed for the systems with $R>1$; a comparable $\tan \delta$ and peak amplitude can be observed. The C-M reprocessed ELO/DTBA $R=0.83$ material is the most affected, as displayed already by the TGA analysis, exhibiting a decrease of the crosslink density from $1.19 \mathrm{mmol} \mathrm{cm} \mathrm{cos}^{-3}$ for the virgin thermoset to $0.79 \mathrm{mmol} \mathrm{cm}^{-3}$. Anyhow, the combined $\mathrm{C}-\mathrm{M}$ reprocessing seems to affect the crosslink density less, in comparison with single mechanical reprocessing where we have found an important decrease in the network density for some systems. Moreover, some C-M reprocessed systems displayed a higher modulus in the rubbery plateau (Fig. S9, ESI $\dagger$ ), exhibiting a higher crosslink density.

ELO/DTBA $R=2$ is less impacted by the C-M recycling, showing almost the same crosslinking density after mechanical or $\mathrm{C}-\mathrm{M}$ reprocessing.

\section{Conclusions}

Thermally and chemically reversible dynamic covalent networks were obtained by crosslinking ELO, ESO and the (ELO + ESO) mixture of epoxidized vegetable oils with a disulfide-containing carboxylic diacid hardener. The dependence of the thermomechanical properties and the reprocessing ability and behaviour of the thermosets on the ratio between epoxide monomer and aromatic diacid hardener was highlighted.

FT-IR analysis confirmed that higher copolymerization conversions were obtained for the systems with a stoichiometric 


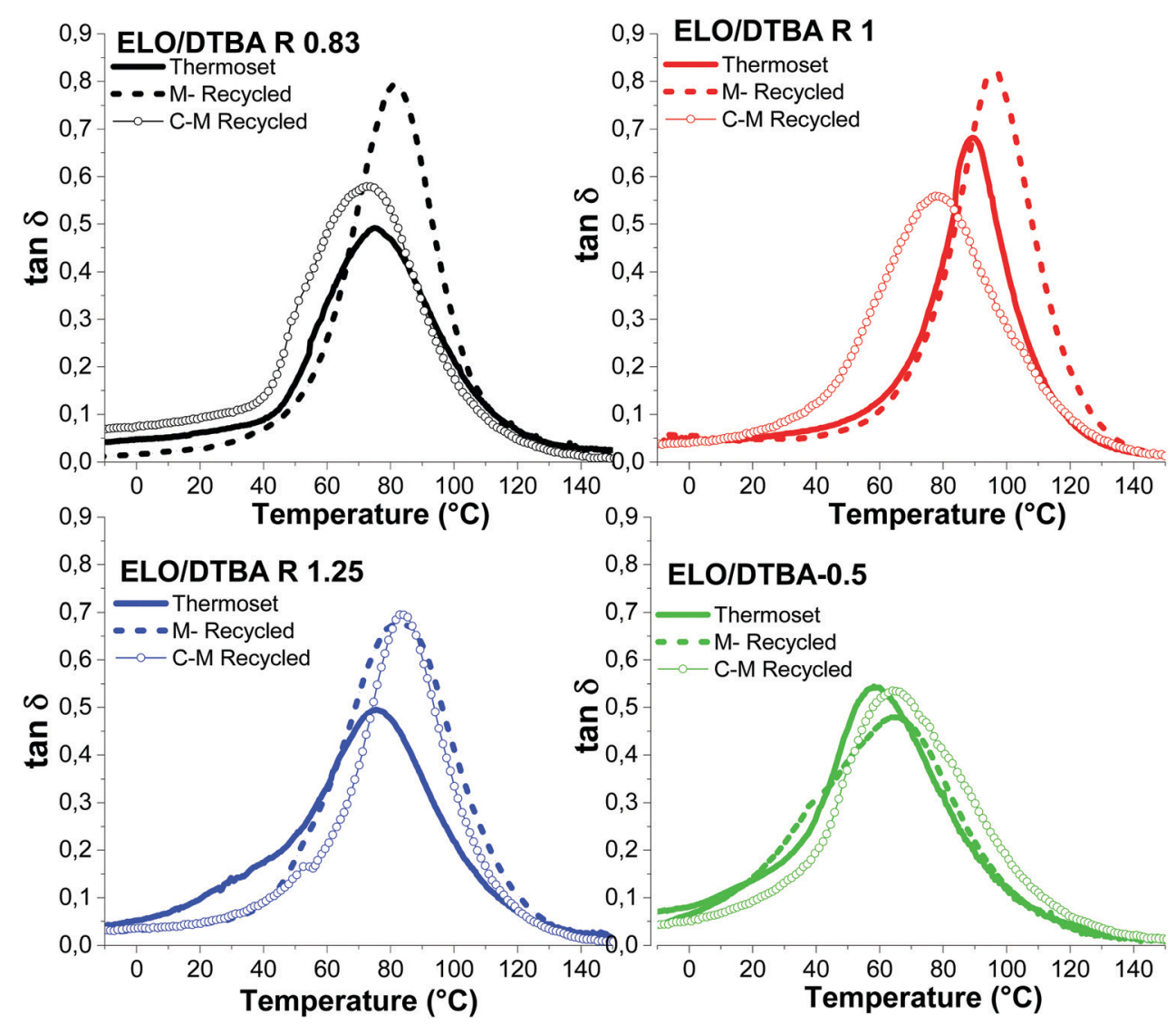

Fig. 10 Comparison of $\tan \delta$ vs. temperature curves for ELO/DTBA systems: virgin, M-reprocessed and C-M recycled and reprocessed materials.

ratio. Thermomechanical analysis showed that the systems out of stoichiometry have lower values of $\tan \delta$ and crosslink densities, in contrast with the thermal stability that increases with a lower crosslinker amount.

The synthesized thermosets showed excellent recycling and reprocessing abilities. The swelling abilities of these thermosets were studied before and after the reprocessing procedure, showing that the reprocessed materials swell more than the virgin resins. Finally, due to the high swelling in THF until dissociation, the thermosets were chemically recycled, dried and reprocessed. The resulting materials were compared with those mechanically reprocessed, showing better properties when $R<1$. Even if the stoichiometric ratio $R=1$ guarantees better mechanical properties, the thermosets cured in defect of hardener showed better resistance and comparable reprocessing ability.

\section{Experimental section}

\section{Materials}

The structures of chemicals used in this study are illustrated in Chart 1 . The vegetable-based epoxy molecules, ELO (epoxidized linseed oil) (a) and ESO (epoxidized soybean oil) (b), were obtained from Valtris Specialty Chemicals.

The hardener and initiator products are commercially available and were used as received without further purification. 2,2'-Dithiodibenzoic acid (DTBA) 95\% (c) and imidazole (IM)
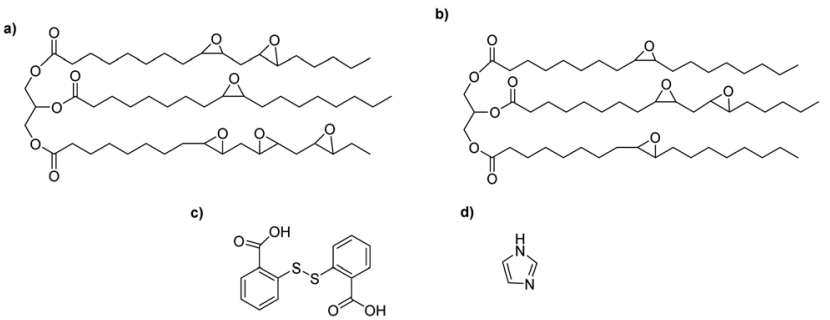

Chart 1 Chemical structure of starting materials: ELO (a), ESO (b), DTBA (c), IM (d).

99\% (d) were purchased from Sigma-Aldrich. The reagent characteristics are summarized in Table S1 (ESI $\dagger$ ).

\section{Sample preparation}

To highlight the influence of epoxy/acid ratio $(R)$ on recyclability, different formulations were prepared from ELO/DTBA, ESO/ DTBA and (ELO + ESO)/DTBA. The epoxy/acid ratio, $R$, was defined by the number of functional groups, so:

$$
R=\frac{\text { epoxy groups }}{\text { acid groups }}
$$

where the acid and epoxy functions are considered to both be monofunctional in the reaction of epoxide and acid. ${ }^{30}$ Therefore, the following $R$ ratios were studied: $R=0.83(\mathrm{e} / \mathrm{a}=1 / 1.2) ; 1$ $(\mathrm{e} / \mathrm{a}=1 / 1) ; 1.25(\mathrm{e} / \mathrm{a}=1 / 0.8) ; 2(\mathrm{e} / \mathrm{a}=1 / 0.5) ;$ and $3.33(\mathrm{e} / \mathrm{a}=1 / 0.3)$. 
The initiator, imidazole, was used at an optimal content of $1 \mathrm{wt} \%$, as previously determined. ${ }^{13}$

To prepare the reactive mixtures, EVO was heated at $80{ }^{\circ} \mathrm{C}$ to decrease its viscosity and thereafter mixed with the initiator and the hardener. The obtained mixtures were placed into silicone moulds and cured in an oven. The curing program was previously studied by DSC analyses of the crosslinking reaction for each system. For all the systems, the curing was performed at temperatures ranging from 130 to $140{ }^{\circ} \mathrm{C}$ for 60 minutes, followed by a post-curing at 170 or $180{ }^{\circ} \mathrm{C}$ for 30 minutes for ELO and ESO resins, respectively.

Two formulations were prepared by the combination of ELO and ESO. Starting from a molar mixture ELO/ESO of $2 / 1$, the following epoxy/acid ratios were prepared: $R=0.83(\mathrm{e} / \mathrm{a}=1 / 1.2)$ and $1(\mathrm{e} / \mathrm{a}=1 / 1)$. To prepare the thermosets, we applied the same curing protocol as for the ESO based systems (Table S2, $\mathrm{ESI} \dagger)$.

\section{Reprocessing protocol}

For the mechanical recycling procedure, a piece of crosslinked thermoset was ground. The obtained powder was compressed between two Kapton films in a CARVER heating press. The applied reprocessing conditions are reported in Table 2 and Table S3 (ESI $\dagger$ ).

\section{Analytical methods}

Differential scanning calorimetry (DSC). DSC measurements were carried out using a Mettler-Toledo DSC 3 instrument controlled by STAR (C) Software developed by Mettler-Toledo. The instrument heat flow and temperature were calibrated at 3 points using water, indium and zinc standards. Samples of 8-12 mg were placed into $40 \mu \mathrm{L}$ aluminium crucibles. The DSC technique was used for the investigation of copolymerization reaction and for the identification of thermoset glass transition using a heating rate $\beta$ of $10{ }^{\circ} \mathrm{C} \min ^{-1}$ over a temperature range from 25 to $210{ }^{\circ} \mathrm{C}$ for the copolymerization study and from -80 to $180{ }^{\circ} \mathrm{C}$ for the $T_{\mathrm{g}}$ analysis.

Fourier-transformed infrared (FT-IR) spectroscopy. The FT-IR spectra of the samples were recorded using a Thermo Scientific Nicolet iS50 FT-IR spectrometer with a deuterated L-alanine doped triglycine sulfate (DLaTGS) detector in attenuated total reflectance (ATR) mode from 500 to $4000 \mathrm{~cm}^{-1}$, at a resolution of $4 \mathrm{~cm}^{-1}$. The decrease of the epoxy and acid peak intensities was followed during the curing reactions, in order to evaluate their conversion. The obtained data were analysed using OMNIC software. The percentage of the copolymerization conversion, determined by this study, is defined by eqn (2):

$$
\%=\frac{\left(\frac{A 823}{A 1586}\right) 0-\left(\frac{A 823}{A 1586}\right) t}{\left(\frac{A 823}{A 1586}\right) 0} \times 100
$$

where the area of absorbance peaks was calculated considering the spectra of unreacted mixtures, at the initial time $\left(A_{0}\right)$, and the spectra of the crosslinked materials after complete curing in an oven $\left(A_{\mathrm{t}}\right)$. The peak at $823 \mathrm{~cm}^{-1}$ was followed,

corresponding to the oxirane $\mathrm{C}-\mathrm{O}$ groups. A reference band was used at $1586 \mathrm{~cm}^{-1}$, belonging to the $\delta_{\mathrm{C}-\mathrm{C}}$ of the aromatic signal from the DTBA hardener.

Thermogravimetric analysis (TGA). TGA measurements were carried out using a Mettler-Toledo TGA 2. The microbalance has a precision of $\pm 0.1 \mu \mathrm{g}$. Samples of about $10 \mathrm{mg}$ were placed into $70 \mu \mathrm{L}$ alumina pans. To characterize the thermal stability of the thermosets, the samples were heated at $10{ }^{\circ} \mathrm{C} \mathrm{min}^{-1}$ from 25 to $1000{ }^{\circ} \mathrm{C}$ under $50 \mathrm{~mL} \mathrm{~min}^{-1}$ air flow.

Dynamic mechanical analysis (DMA). DMA was performed using a Mettler-Toledo DMA 1 instrument, equipped with STAR (C) software. The analysed samples had rectangular dimensions of $30 \times 7 \times 2 \mathrm{~mm}^{3}$ (length $\times$ width $\times$ thickness). Each system was analysed 3 times and the values were averaged. DMA was performed in temperature-scanning mode with a constant displacement amplitude and frequency using the tension method for the analysis of virgin and recycled materials. Elastic modulus $\left(E^{\prime}\right)$ and damping factor $(\tan \delta)$ were collected at a $3{ }^{\circ} \mathrm{C} \min ^{-1}$ heating rate from -20 to $150{ }^{\circ} \mathrm{C}$ and $1.0 \mathrm{~Hz}$ frequency. The glass transition temperature was assigned at the maximum of the damping factor $\left(\tan \delta=E^{\prime \prime} / E^{\prime}\right)$.

Crosslinking density was calculated by using eqn (3):

$$
\nu=\frac{E^{\prime}}{3 R T}
$$

where $E^{\prime}$ is the storage modulus of the thermoset in the rubbery plateau region at $T_{\mathrm{g}}+50{ }^{\circ} \mathrm{C}, R$ is the gas constant and $T$ is the absolute temperature in Kelvin.

\section{Solvent stability}

Thermoset solvent stability was studied in ethanol, methanol, DMSO, acetone, toluene, water, DMF, DCM, THF and toluene for 48 hours at room temperature.

Toluene was selected as the solvent for the swelling experiments. These experiments were performed for the virgin and reprocessed resins. The swelling ratio $Q$ can be defined by eqn (4):

$$
Q=\frac{m_{\mathrm{s}}-m_{\mathrm{u}}}{m_{\mathrm{u}}}
$$

where $m_{\mathrm{s}}$ and $m_{\mathrm{u}}$ are the swollen and unswollen masses of the sample, respectively.

All the solvent tests were conducted on rectangular specimens of $10 \times 10 \times 2 \mathrm{~mm}^{3}$ dimensions.

\section{Abbreviations}

$\mathrm{VO}$

EVO

ELO

ESO

IM

DTBA

DGEBA

DCA

DMF

DCM
Vegetable oil

Epoxidized vegetable oil

Epoxidized linseed oil

Epoxidized soybean oil

Imidazole

2,2'-Dithiodibenzoic acid

Diglycidyl ether of bisphenol A

Dicarboxylic acids

$\mathrm{N}, \mathrm{N}$-Dimethylformamide

Dichloromethane 
FT-IR Fourier transform infrared spectroscopy

DSC Differential scanning calorimetry

DMA Dynamic mechanical analysis

TGA Thermogravimetric analysis

DTG Derivative thermogravimetric analysis

THF Tetrahydrofuran

\section{Conflicts of interest}

There are no conflicts to declare.

\section{Acknowledgements}

This work was supported by the ECOXY project funded by the Bio Based Industries Joint Undertaking under the European Union Horizon 2020 research and innovation program (Grant agreement no. 744311).

\section{Notes and references}

1 N. Karak, Vegetable oil-based polymers: properties, processing and applications. Elsevier: 2012.

2 Z. Petrović, Polymers from biological oils, Contemp. Biomater., 2010, 1(1), 39-50.

3 A. E. Gerbase, C. L. Petzhold and A. P. O. Costa, Dynamic mechanical and thermal behavior of epoxy resins based on soybean oil, J. Am. Oil Chem. Soc., 2002, 79(8), 797-802.

4 F. I. Altuna, L. H. Espósito, R. A. Ruseckaite and P. M. Stefani, Thermal and mechanical properties of anhydride-cured epoxy resins with different contents of biobased epoxidized soybean oil, J. Appl. Polym. Sci., 2011, 120(2), 789-798.

5 M. Shibata, N. Teramoto and K. Makino, Preparation and properties of biocomposites composed of epoxidized soybean oil, tannic acid, and microfibrillated cellulose, J. Appl. Polym. Sci., 2011, 120(1), 273-278.

6 H. P. Benecke, B. R. Vijayendran and J. D. Elhard, Plasticizers derived from vegetable oils, US Pat., 6797753, 2004.

7 F. Jaillet, M. Desroches, R. Auvergne, B. Boutevin and S. Caillol, New biobased carboxylic acid hardeners for epoxy resins, Eur. J. Lipid Sci. Technol., 2013, 115(6), 698-708.

8 C. Ding, P. S. Shuttleworth, S. Makin, J. H. Clark and A. S. Matharu, New insights into the curing of epoxidized linseed oil with dicarboxylic acids, Green Chem., 2015, 17(7), 4000-4008.

9 R.-T. Zeng, Y. Wu, Y.-D. Li, M. Wang and J.-B. Zeng, Curing behavior of epoxidized soybean oil with biobased dicarboxylic acids, Polym. Test., 2017, 57, 281-287.

10 S. Ma, C. S. Kovash and D. C. Webster, Effect of solvents on the curing and properties of fully bio-based thermosets for coatings, J. Coat. Technol. Res., 2017, 14(2), 367-375.

11 C. Ding, G. Tian and A. Matharu, Adipic acid - glutaric anhydride - epoxidised linseed oil biobased thermosets with tunable properties, Mater. Today Commun., 2016, 7, 51-58.
12 G. Falco, N. Sbirrazzuoli and A. Mija, Biomass derived epoxy systems: From reactivity to final properties, Mater. Today Commun., 2019, 21, 100683.

13 T.-N. Tran, C. Di Mauro, A. Graillot and A. Mija, Chemical Reactivity and the Influence of Initiators on the Epoxidized Vegetable Oil/Dicarboxylic Acid System, Macromolecules, 2020, 53(7), 2526-2538.

14 F. I. Altuna, V. Pettarin and R. J. J. Williams, Self-healable polymer networks based on the cross-linking of epoxidised soybean oil by an aqueous citric acid solution, Green Chem., 2013, 15(12), 3360-3366.

15 D. Montarnal, M. Capelot, F. Tournilhac and L. Leibler, Silica-Like Malleable Materials from Permanent Organic Networks, Science, 2011, 334(6058), 965.

16 A. Rekondo, R. Martin, A. Ruiz de luzuriaga, G. Cabañero, H. J. Grande and I. Odriozola, Catalyst-free room-temperature self-healing elastomers based on aromatic disulfide metathesis, Mater. Horiz., 2013, Vol. 1, 237.

17 S. Nevejans, N. Ballard, J. I. Miranda, B. Reck and J. M. Asua, The underlying mechanisms for self-healing of poly(disulfide)s, Phys. Chem. Chem. Phys., 2016, 18(39), 27577-27583.

18 X. Jian, Y. Hu, W. Zhou and L. Xiao, Self-healing polyurethane based on disulfide bond and hydrogen bond, 2017, vol. 29.

19 R. Martin, A. Rekondo, A. Ruiz de Luzuriaga, G. Cabañero, H. J. Grande and I. Odriozola, The processability of a poly(ureaurethane) elastomer reversibly crosslinked with aromatic disulfide bridges, J. Mater. Chem. A, 2014, 2(16), 5710-5715.

20 Z. Ma, Y. Wang, J. Zhu, J. Yu and Z. Hu, Bio-based epoxy vitrimers: Reprocessibility, controllable shape memory, and degradability, J. Polym. Sci., Part A: Polym. Chem., 2017, 55(10), 1790-1799.

21 C. Di Mauro, T.-N. Tran, A. Graillot and A. Mija, Enhancing the Recyclability of a Vegetable Oil-Based Epoxy Thermoset through Initiator Influence, ACS Sustainable Chem. Eng., 2020, 8(20), 7690-7700.

22 L. Imbernon, E. K. Oikonomou, S. Norvez and L. Leibler, Chemically crosslinked yet reprocessable epoxidized natural rubber via thermo-activated disulfide rearrangements, Polym. Chem., 2015, 6(23), 4271-4278.

23 S. Ma and D. Webster, Naturally Occurring Acids as CrossLinkers To Yield VOC-Free, High-Performance, Fully BioBased, Degradable Thermosets, Macromolecules, 2015, 48, 7127-7137.

24 N. Boquillon and C. Fringant, Polymer networks derived from curing of epoxidised linseed oil: influence of different catalysts and anhydride hardeners, Polymer, 2000, 41(24), 8603-8613.

25 C. D. Wingard and C. L. Beatty, Crosslinking of an epoxy with a mixed amine as a function of stoichiometry. II. Final properties via dynamic mechanical spectroscopy, J. Appl. Polym. Sci., 1990, 41(11-12), 2539-2554.

26 J. Galy, A. Sabra and J.-P. Pascault, Characterization of epoxy thermosetting systems by differential scanning calorimetry, Polym. Eng. Sci., 1986, 26(21), 1514-1523.

27 T. S. Omonov and J. M. Curtis, Biobased epoxy resin from canola oil, J. Appl. Polym. Sci., 2014, 131, 8. 
28 A. Jurowska and K. Jurowski, Vitrimers - The miracle polymer materials combining the properties of glass and plastic?, Chemik, 2015, 69, 392-394.

29 M. Chen, L. Zhou, Y. Wu, X. Zhao and Y. Zhang, Rapid Stress Relaxation and Moderate Temperature of Malleability Enabled by the Synergy of Disulfide Metathesis and
Carboxylate Transesterification in Epoxy Vitrimers, ACS Macro Lett., 2019, 8(3), 255-260.

30 L. Matějka, S. Pokorný and K. Dušek, Acid curing of epoxy resins. A comparison between the polymerization of diepoxidediacid and monoepoxide-cyclic anhydride systems, Die Makromolekulare Chemie, 1985, 186(10), 2025-2036. 